\title{
Informed consent and ethical re-use of African genomic data
}

\author{
Galen EB Wright ${ }^{1}$, Adebowale A Adeyemo $^{2}$ and Nicki Tiffin ${ }^{1 *}$
}

\begin{abstract}
Rapid advances in human genomic research are increasing the availability of genomic data for secondary analysis. Particularly in the case of vulnerable African populations, ethics and informed consent processes need to be transparent-both to ensure participant protection, as well as to share skills and to evolve best practice for informed consent from a shared knowledge base. An open dialogue between all stakeholders can facilitate this.
\end{abstract}

Keywords: Informed consent, Secondary use, Ethics, Africa

\section{Ethical re-use of African genomic data}

The health genomics revolution is well under way, thanks to the increased accessibility of rapidly advancing sequencing technologies [1]. Africa is primed to enter this arena, especially given significant new research support from the Human Heredity and Health in Africa (H3Africa) Initiative [2] funded by the National Institutes of Health (NIH, USA) and the Wellcome Trust (UK) [3]. Within Africa, the support from this initiative is unprecedented in size and scope, currently including eight consortia/collaborative centers, eight research projects, four biorepository projects and a continent-wide bioinformatics network. Genomic data-sharing and secondary use is the key to accelerating discovery and many initiatives such as the Global Alliance for Genomics and Health [4], and the H3Africa Bioinformatics Network [5] are enabling data-sharing. This must, however, be realised within a framework of high ethical standards and truly informed participant consent [6-8].

Consent for secondary use of data and samples is sensitive within Africa, for various reasons: localised cultural sensitivities, and the vast cultural and ethnic diversity across African populations, are not always appreciated by external researchers [9]; many African populations are vulnerable to exploitation because of low education levels, and poor access to health care may create dependence on study inclusion for access

\footnotetext{
* Correspondence: nicki@sanbi.ac.za

'South African National Bioinformatics Institute/Medical Research Council of South Africa Bioinformatics Unit, University of the Western Cape, Private Bag X17, Bellville 7535, South Africa

Full list of author information is available at the end of the article
}

to care $[10,11]$; and African governments, institutes, and researchers are wary of repeating the historical outflow of samples and data from the continent through collaborations outside Africa [12,13].

Institutional and governmental ethics review boards (IRBs) are currently the gate-keepers for re-use of African genomic data and for validating informed consent processes. There are many excellent IRBs and ethicists in Africa, but they are often under-resourced (for example, see [14] and [15]). They may lack capacity to train members in evaluating human genomics research, e.g. issues with genomic data de-identification,-a conceptual paradigm shift away from existing clinical and genetic data that do not encapsulate the individual as a whole. There is, therefore, a strong need in Africa for ethics skills development [16], sharing of ethics and informed consent experiences, and building on current practice. Ensuring robust informed consent processes is crucial for protection of African research participants-particularly because once data leaves Africa under secondary use consent, it moves beyond the jurisdiction of the country of origin and participant protection becomes reliant on the host country.

To date, we have found it surprisingly difficult to access informed consent templates, standard operating procedures and 'information for participants' from published African genomic studies [13]. Reluctance to share ethics and informed consent template documentation may arise from concerns around privacy and legal implications for both IRBs and researchers. In the absence of such prior information, each new genomic study often has to develop 
anew the informed consent process, templates, and forms without being able to learn from previous studies. Through this continual reinvention of the informed consent process, we fail to grow our understanding of beneficence and risks for African research participants or to improve these processes incrementally with each new study.

Issues around data re-use and informed consent may raise concerns about secondary use of participant genomic data. Currently, a statement about ethics approval and informed consent is usually provided in publications and is often considered sufficient for secondary use access to study data obtained through correspondence with the authors. In some cases, data access committees may review proposals for secondary use of genomic data (for example, see [17]), but the original ethics documentation and informed consent templates are seldom available for scrutiny by downstream data users to ensure that participant consent was fairly and ethically obtained for their own secondary analysis. Similarly, except for some large-scale projects of human genetic variation [8], such resources are seldom available for reference by researchers developing their own best practice for informed consent.

These issues raise questions around transparency and skills-sharing to develop the best practice for ethics, informed consent and participant protection particularly in Africa-although they are pertinent for all health genomics research.

Firstly, can journals that publish genomic research involving human subjects encourage more transparency through submission of ethics-related documentation and templates when accepting manuscripts for publication? This might take the form of providing the option to submit ethics documentation templates (such as informed consent templates) for some studies or encouragement to provide such documentation as supplementary data where authors are willing to do so. Secondly, can public databases for human genome data facilitate transparency around ethics and informed consent processes? Full ethics documentation could be supplied at the time of data submission and could be provided to researchers requesting access to the data through appropriate committees. Thirdly, should the human genomic research community explore ways (e.g. through centralized databases) to share templates for informed consent and patient information in the interests of ethics skills-sharing and developing best practices for participant protection? Such resources could assist researchers in developing appropriate ethics templates for their own studies (but should not be seen as developing a 'one-size-fits-all' approach to informed consent protocols).

Whilst the answers remain unclear, there is a need for open dialogue between stakeholders about maintaining and supporting sustainable ethical compliance and integrity when dealing with human genomic data. As long as economic and participant vulnerability for African populations remains, these issues are particularly pertinent when dealing with data originating from Africa.

\section{Abbreviations}

H3Africa: Human Heredity and Health in Africa; H3ABioNet: H3Africa bioinformatics network; IRB: institutional review board; NIH: National Institutes of Health.

\section{Competing interests}

The authors declare that they have no competing interests.

\section{Authors' contributions}

The authors all planned, wrote and reviewed the manuscript. All authors read and approved the final manuscript.

\section{Acknowledgements}

We acknowledge Professor Marc Blockman (University of Cape Town) and the University of Cape Town Ethics Review Board for discussion of manuscript contents. NT is funded by the Medical Research Council of South Africa, the H3Africa Kidney Disease Research Network (National Institutes of Health, grant number 1U54HG006939) and the H3Africa Bioinformatics Network (National Institutes of Health Common Fund, grant number U41HG006941). AA is supported by the National Institutes of Health Intramural Research Program in the Center for Research on Genomics and Global Health (CRGGH). The CRGGH is supported by funds from the Office of the Director, NIDDK and NHGRI at National Institutes of Health (Z01HG200362). GW was funded by the South African National Research Foundation. The content of this publication is solely the responsibility of the authors and does not necessarily represent the official views of the National Institutes of Health, the Medical Research Council of South Africa or the National Research Foundation of South Africa.

\section{Author details}

${ }^{1}$ South African National Bioinformatics Institute/Medical Research Council of South Africa Bioinformatics Unit, University of the Western Cape, Private Bag X17, Bellville 7535, South Africa. ${ }^{2}$ National Human Genome Research Institute, National Institutes of Health, Building 12A, Room 4047, 12 South Drive, MSC 5635, Bethesda, MD 20892-5635, USA.

Received: 5 August 2014 Accepted: 11 October 2014

Published online: 22 October 2014

\section{References}

1. Green ED, Guyer MS, Manolio TA, Peterson JL: Charting a course for genomic medicine from base pairs to bedside. Nature 2011, 470:204-213.

2. Human Heredity and Health in Africa (H3Africa) Initiative. [www.H3Africa.org]

3. H3Africa Consortium: Research capacity. Enabling the genomic revolution in Africa. Science 2014, 344:1346-1348.

4. Global Alliance for Genomics and Health. [http://genomicsandhealth.org/] 5. H3Africa Bioinformatics Network. [www.H3ABioNet.org]

6. McEwen JE, Boyer JT, Sun KY: Evolving approaches to the ethical management of genomic data. Trends Genet TIG 2013, 29:375-382.

7. Kaye J, Heeney C, Hawkins N, de Vries J, Boddington P: Data sharing in genomics-re-shaping scientific practice. Nat Rev Genet 2009, 10:331-335.

8. Institute of Medicine (US) Committee on the Role of Institutional Review Boards in Health Services Research Data Privacy Protection: Protecting Data Privacy in Health Services Research. Washington (DC): National Academies Press (US); 2000

9. Sampson DA, Caldwell D, Taylor AD, Taylor JY: Blending genetics and sociocultural historical inquiry: ethics, culture, and human subjects protection in international cross cultural research. Yale J Biol Med 2013, 86:89-98.

10. Lema VM, Mbondo M, Kamau EM: Informed consent for clinical trials: a review. East Afr Med J 2009, 86:133-142.

11. Paré Toe L, Ravinetto RM, Dierickx S, Gryseels C, Tinto H, Rouamba N, Diallo I, Cissao Y, Bayala K, Hausmann S, Muela J, D’Alessandro U, Peeters Grietens K: Could the decision of trial participation precede the informed consent process? Evidence Burkina Faso PloS One 2013, 8:e80800. 
12. De Vries J, Bull SJ, Doumbo O, Ibrahim M, Mercereau-Puijalon O, Kwiatkowski D, Parker M: Ethical issues in human genomics research in developing countries. BMC Med Ethics 2011, 12:5.

13. Wright GE, Koornhof $P G$, Adeyemo AA, Tiffin N: Ethical and legal implications of whole genome and whole exome sequencing in African populations. BMC Med Ethics 2013, 14:21.

14. Oyibo WA, Krugher M, Fagbenro-Beyioku AF: The roles, challenges and institutionalization of institutional review boards. Niger Q J Hosp Med 2008, 18:115-119.

15. Ramsay M, de Vries J, Soodyall H, Norris SA, Sankoh O: Ethical issues in genomic research on the African continent: experiences and challenges to ethics review committees. Hum Genomics 2014, 8:15.

16. Nyika A, Kilama W, Chilengi R, Tangwa G, Tindana P, Ndebele P, Ikingura J: Composition, training needs and independence of ethics review committees across Africa: are the gate-keepers rising to the emerging challenges? J Med Ethics 2009, 35:189-193.

17. Network MGE: A global network for investigating the genomic epidemiology of malaria. Nature 2008, 456:732-737.

doi:10.1186/s40246-014-0018-7

Cite this article as: Wright et al:: Informed consent and ethical re-use of

African genomic data. Human Genomics 2014 8:18.

\section{Submit your next manuscript to BioMed Central and take full advantage of:}

- Convenient online submission

- Thorough peer review

- No space constraints or color figure charges

- Immediate publication on acceptance

- Inclusion in PubMed, CAS, Scopus and Google Scholar

- Research which is freely available for redistribution 